\title{
EXISTENCE AND STABILITY ANALYSIS BY FIXED \\ POINT THEOREMS FOR A CLASS OF NON-LINEAR CAPUTO FRACTIONAL DIFFERENTIAL EQUATIONS
}

\author{
ARJUMAND SEEMAB ${ }^{1}$ AND MUJEEB UR REHMAN ${ }^{2}$ \\ ${ }^{1,2}$ School of Natural Sciences \\ National University of Sciences and Technology \\ Islamabad, PAKISTAN
}

\begin{abstract}
The main purpose of this work is to establish existence result and stability criteria for a class of fractional order differential equations using fixed point theorems. Existence results are based on Schauder's fixed point theorem, Banach contraction principle and, emphasis is put on the application of the Krasnoselskii's fixed point theorem to establish stability criteria of a specific class of fractional order differential equations. An example is given to show the usefulness of the stability result.
\end{abstract}

AMS Subject Classification: 26A33

Key Words: fractional differential equations, Caputo derivative, Mittag-Leffler function, stability

Received: August 2017,31; Accepted: April 12, 2018;

Published: June 7, $2018 \quad$ doi: 10.12732/dsa.v27i3.1

Dynamic Publishers, Inc., Acad. Publishers, Ltd. https://acadsol.eu/dsa

\section{INTRODUCTION}

The initiative idea of non-integer order derivatives is quite old and history of fractional calculus spans on three centuries. Since in the mid twentieth century, and latter decades the number of papers devoted to fractional calculus increased rapidly. One of the reasons for the significant interest in the field of fractional calculus is that verity of physical [1], chemical [2] and biological [3] phenomena can be described with fractional differential equations. The field of fractional calculus can be considered 
as new branch of applied mathematics. A fair amount of basic mathematical theory related to the study of fractional calculus attributed to Leibniz, Caputo, Liouville, Riemann, Euler and many others. However, in the past few decades more and more convincing applications in different fields of engineering and sciences [4] have been found. It is notable that a larger part of research work is committed to the existence theory of fractional differential equations(FDEs)(see $[5,6,7])$. Recently, many researchers used fractional differential equations as a valuable tool in modeling of various stable physical phenomena. However, exploration of stability theory of nonlinear FDEs is still in its initial stages and a bunch of work could be done in this area. A little while back the theory of FDEs has been investigated enormously and several fundamental results are obtained which includes the stability theory as well. In mathematical language, stability theory discusses the convergence of solutions of differential equations under the small changes in the initial data. The question of stability is a central task in the study of FDEs and it has been studied by many authors (see $[8,9,10,11,12,13,14,15,16])$. Anyhow, the analysis for stability of nonlinear FDEs is relatively more tricky than that of classical integer order differential equations. Partially this is because the fractional derivatives are nonlocal and inherent a weakly singular kernels. During the last decades, many researchers have been attracted to the study of stability theory of non-linear fractional differential equations and therefore various methods are introduced. However, we note that only a few steps are taken to use the fixed point theorems in the investigation of the stability of FDEs. The application of fixed point theorem to study the stability properties of differential equations have extensively been studied by T. A. Burton (see [17] and references therein). In [18], Fudong Ge and Chunhai Kou considered the stability of Caputo type FDEs for $\alpha \in(1,2)$. They used Krasnoselskii's fixed point theorem to obtain their main results. Motivated by their work, in this paper we present sufficient conditions for the existence of solution in conjunction with stability analysis of the following class of non-linear fractional initial value problem

$$
{ }^{c} D_{0, t}^{\alpha} u(t)+u(t)=a(t) u(t)+f(t, u(t)), \quad u(0)=u_{0}, \quad 0<\alpha \leq 1,
$$

where $u_{0} \in \mathbb{R}, f:[0, \infty) \times \mathbb{R} \rightarrow \mathbb{R}$ and $a:[0, \infty) \rightarrow \mathbb{R}$ are continuous functions and $f(t, 0) \equiv 0$. The differential operator ${ }^{c} D_{0, t}^{\alpha}$ is the standard Caputo fractional differential operator of order $\alpha \in(0,1]$.

The tools used in this paper remain the same as in $[18,17]$. A crucial difference here in this work and in references cited above is that we are using an equivalent integral equation for (1.1) which is derived by the Laplace transform. This approach allows us to use properties of Mittage-Lefler function to simplify calculations while establishing stability results. This approach has been used by [21] to study stability of system of fractional differential equations. To the best of authors knowledge, this approach has never been used to study the stability via fixed point approach. 
We arranged this paper as follows: Section 2 contains some basic definitions and lemmas that are helpful in what follows, and our main results are presented in section 3.

\section{PRELIMINARIES}

For ease, this section is devoted to providing an outline of few ideas, definitions and some fundamental outcomes from fractional calculus which are utilized throughout this article.

Definition 2.1. [19] The Caputo derivative ${ }^{c} D_{a, t}^{\alpha}$ of fractional order $\alpha \in[0, \infty)$ of function $u \in C^{m+1}[a, b]$, is defined as

$$
{ }^{c} D_{a, t}^{\alpha} u(t)=I_{a}^{m-\alpha} D^{m} u(t)=\int_{a}^{t} \frac{(t-\tau)^{m-\alpha-1}}{\Gamma(m-\alpha)} u^{(m)}(\tau) d \tau,
$$

where $m-1<\alpha \leq m \in \mathbb{Z}^{+}$and $I_{a}^{\alpha} u(t):=\int_{a}^{t} \frac{(t-\tau)^{\alpha-1}}{\Gamma(\alpha)} u(\tau) d \tau$ is the Riemann-Liouvill integral of fractional order $\alpha \in(0, \infty)$.

The Mittag-Leffler (ML) function is simple generalization of exponential function introduced by Swedish mathematician Gosta Mittag-Leffler (1846-1927). It plays significant role in qualitative and quantitative theory of fractional differential equations.

Definition 2.2. The two parametric ML function $E_{\alpha, \beta}(t)$ is defined as

$$
E_{\alpha, \beta}(t)=\sum_{k=0}^{\infty} \frac{t^{k}}{\Gamma(\alpha k+\beta)} \quad t \in \mathbb{R}, \quad \alpha, \beta>0 .
$$

From numerical evidences, as illustrated in Figure [20] we notice that, for $t \in$ $[0, \infty), 0<\alpha \leq 1$, the one-parameter Mittag-Leffler function $E_{\alpha, 1}\left(-t^{\alpha}\right)$ is decreasing function of $t$ and it is bounded from above by 1 . That is $E_{\alpha, 1}\left(-t^{\alpha}\right) \leq 1$. Furthermore, it is to be noted that

$$
\lim _{t \rightarrow \infty} E_{\alpha, 1}\left(-t^{\alpha}\right)=0
$$

Now, we present definition for stability of solutions for (1.1).

Definition 2.3. The solution $u=\psi(t)$ of $(1.1)$ is called

(i) stable, if for every $\epsilon>0$ and $t_{0} \geq 0$, there exists a $\delta=\delta\left(t_{0}, \epsilon\right)>0$ such that $\left|u\left(t, u_{0}, t_{0}\right)-\psi(t)\right|<\epsilon$ for $\left|u_{0}-\psi\left(t_{0}\right)\right| \leq \delta\left(t_{0}, \epsilon\right)$ and all $t \geq t_{0} ;$

(ii) attractive, if there exists $\sigma\left(t_{0}\right)>0$ such that $\left\|u_{0}\right\|_{\infty} \leq \sigma$ implies $\lim _{t \rightarrow \infty} u\left(t, u_{0}, t_{0}\right)=$ 0 


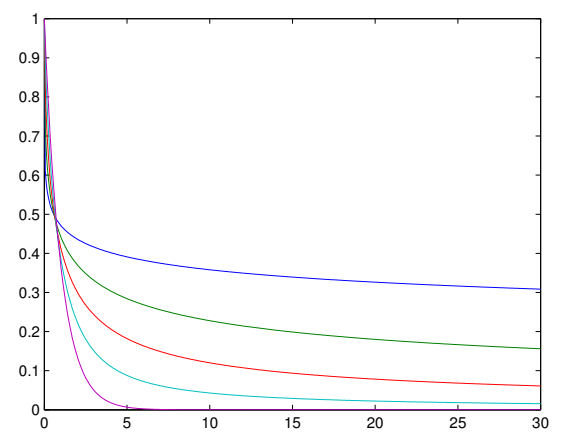

Figure 1: Graphs of $E_{\alpha, 1}\left(-t^{\alpha}\right)$ for $\alpha=0.2,0.4,0.6,0.8,1$.

(iii) asymptotically stable if it is stable and attractive.

To continue promote, we give the accompanying auxiliary lemma.

Lemma 2.4. [21] Let $0<\alpha \leq 1$ and $u_{0} \in \mathbb{R}$. Moreover assume that $f:[0, \infty) \rightarrow$ $\mathbb{R}, a:[0, \infty) \rightarrow \mathbb{R}$ are continuous and $f(t, 0) \equiv 0$. Then $u(t) \in C[\mathbb{R}, \mathbb{R}]$ is a solution of (1.1) if and only if it is the solution of the integral equation

$$
\begin{aligned}
u(t)=u_{0} E_{\alpha, 1}\left(-t^{\alpha}\right)+\int_{0}^{t} E_{\alpha, 1}\left(-(t-\tau)^{\alpha}\right) & a(\tau) u(\tau) d \tau \\
& +\int_{0}^{t} E_{\alpha, 1}\left(-(t-\tau)^{\alpha}\right) f(\tau, u(\tau)) d \tau
\end{aligned}
$$

\section{MAIN RESULTS}

In this section, we prove results for the existence and stability of the solutions of (1.1).

We begin with some notations for our next theorems.

Assume

$\left(H_{1}\right) \sup _{t \geq 0} \int_{0}^{t} a(\tau) d \tau \leq \rho_{2}<1-\rho_{1}, \rho_{1} \in(0,1)$.

Let $\mathcal{B}:=\left\{u(t) \in C\left(\left[0, \chi_{1}\right], \mathbb{R}\right):\|u\|_{\infty}<\infty\right\}$, where $\chi_{1}=\frac{\rho_{1} \epsilon}{\|f\|_{\infty}}, \epsilon>0$. And $\mathcal{B}$ is a Banach space equipped with the norm $\|u\|_{\infty}=\sup _{t \in[0, T]}|u(t)|, T>0$. Furthermore, for any $t \geq 0$, we assume $\|\phi\|_{t}=\max \{|\phi(t)|: 0 \leq s \leq t\}$, for any given $\phi \in C\left[0, \chi_{1}\right]$ and suppose $\mathcal{M}(\epsilon)=\left\{u: u \in \mathcal{B},\|u\|_{\infty} \leq \epsilon\right\}, \epsilon>0$.

Theorem 3.1. Consider the integral equation (2.3), where $f:[0, \infty) \times \mathbb{R} \rightarrow \mathbb{R}$, is a bounded and continuous function on $\mathbb{R}$, that satisfies the Lipschitz condition

$$
\|f(t, u)-f(t, v)\|_{\infty} \leq \mathcal{L}\|u-v\|_{\infty}, \text { for all } t \in[0, T], T>0,
$$


where $\mathcal{L}$ is a positive constant. Then there exist at least one solution of (1.1).

Proof. Let us set $\Lambda \geq \frac{\left|u_{0}\right|+r T}{1-\mathcal{M} T}, \mathcal{M}<\frac{1}{T}$ where $\mathcal{M}=\sup _{t \in[0, T]}\{a(t)\}$. Consider the non-empty closed convex subset $\mathcal{K}=\left\{u: u \in \mathcal{B},\|u\|_{\infty} \leq \Lambda\right\}$ of $\mathcal{B}$. We consider the mapping $\mathcal{F}$ on $\mathcal{K}$ as defined in equation (3.1).

Also let $\|f(t, u)\|_{\infty} \leq r \forall(t, u) \in[0, T] \times \mathbb{R}$.

First we show that $\mathcal{F}$ maps $\mathcal{K}$ into $\mathcal{K}$.

$$
\begin{aligned}
|(\mathcal{F} u)(t)|= & \mid u_{0} E_{\alpha, 1}\left(-t^{\alpha}\right)+\int_{0}^{t} E_{\alpha, 1}\left(-(t-\tau)^{\alpha}\right) f(\tau, u(\tau)) d \tau \\
& +\int_{0}^{t} E_{\alpha, 1}\left(-(t-\tau)^{\alpha}\right) a(\tau) u(\tau) d \tau \mid \\
\leq & \left|u_{0}\right|+(\Lambda \mathcal{M}+r) T \leq \Lambda
\end{aligned}
$$

Now we prove that $\mathcal{F}(\mathcal{B})$ is relatively compact.

Taking $0 \leq t_{1} \leq t_{2} \leq T$, we have

$$
\begin{aligned}
& \left|(\mathcal{F} u)\left(t_{1}\right)-(\mathcal{F} u)\left(t_{2}\right)\right|=\mid u_{0} E_{\alpha, 1}\left(-t_{1}^{\alpha}\right)+\int_{0}^{t_{1}} E_{\alpha, 1}\left(-\left(t_{1}-\tau\right)^{\alpha}\right) f(\tau, u(\tau)) d \tau \\
& \quad+\int_{0}^{t_{1}} E_{\alpha, 1}\left(-\left(t_{1}-\tau\right)^{\alpha}\right) a(\tau) u(\tau) d \tau-u_{0} E_{\alpha, 1}\left(-t_{2}^{\alpha}\right) \\
& \quad-\int_{0}^{t_{2}} E_{\alpha, 1}\left(-\left(t_{2}-\tau\right)^{\alpha}\right) f(\tau, u(\tau)) d \tau-\int_{0}^{t_{2}} E_{\alpha, 1}\left(-\left(t_{2}-\tau\right)^{\alpha}\right) a(\tau) u(\tau) d \tau \mid \\
& \leq\left|u_{0}\left(E_{\alpha, 1}\left(-t_{1}^{\alpha}\right)-E_{\alpha, 1}\left(-t_{2}^{\alpha}\right)\right)\right| \\
& \quad+\int_{0}^{t_{1}} \mid\left(E_{\alpha, 1}\left(-\left(t_{1}-\tau\right)^{\alpha}\right)-E_{\alpha, 1}\left(-\left(t_{2}-\tau\right)^{\alpha}\right)|| f(\tau, u(\tau)) \mid d \tau\right. \\
& \quad+\int_{t_{1}}^{t_{2}}|f(\tau, u(\tau))| d \tau+\int_{0}^{t_{1}} \mid\left(E_{\alpha, 1}\left(-\left(t_{1}-\tau\right)^{\alpha}\right)-E_{\alpha, 1}\left(-\left(t_{2}-\tau\right)^{\alpha}\right) \mid\right. \\
& \quad \times|a(\tau) u(\tau)| d \tau+\Lambda \int_{t_{1}}^{t_{2}} a(\tau) d \tau \\
& \leq\left|u_{0}\left(E_{\alpha, 1}\left(-t_{1}^{\alpha}\right)-E_{\alpha, 1}\left(-t_{2}^{\alpha}\right)\right)\right| \\
& \quad+\int_{0}^{t_{1}} \mid\left(E_{\alpha, 1}\left(-\left(t_{1}-\tau\right)^{\alpha}\right)-E_{\alpha, 1}\left(-\left(t_{2}-\tau\right)^{\alpha}\right)|| f(\tau, u(\tau)) \mid d \tau\right. \\
& \quad+\int_{0}^{t_{1}} \mid\left(E_{\alpha, 1}\left(-\left(t_{1}-\tau\right)^{\alpha}\right)-E_{\alpha, 1}\left(-\left(t_{2}-\tau\right)^{\alpha}\right)|| a(\tau) u(\tau) \mid d \tau\right. \\
& \quad+(\Lambda \mathcal{M}+r)\left(t_{2}-t_{1}\right) \rightarrow 0
\end{aligned}
$$

as $t_{1} \rightarrow t_{2}$. Now let $u, \widetilde{u} \in \mathcal{K}$ such that

$$
|(\mathcal{F} u)(t)-(\mathcal{F} \widetilde{u})(t)|=\mid \int_{0}^{t} E_{\alpha, 1}\left(-(t-\tau)^{\alpha}\right) f(\tau, u(\tau)) d \tau
$$




$$
\begin{aligned}
& +\int_{0}^{t} E_{\alpha, 1}\left(-(t-\tau)^{\alpha}\right) a(\tau) u(\tau) d \tau \\
& -\int_{0}^{t} E_{\alpha, 1}\left(-(t-\tau)^{\alpha}\right) f(\tau, \widetilde{u}(\tau)) d \tau-\int_{0}^{t} E_{\alpha, 1}\left(-(t-\tau)^{\alpha}\right) a(\tau) \widetilde{u}(\tau) d \tau \mid \\
\leq & \left|\int_{0}^{t} E_{\alpha, 1}\left(-(t-\tau)^{\alpha}\right) a(\tau)(u(\tau)-\widetilde{u}(\tau)) d \tau\right| \\
& +\left|\int_{0}^{t} E_{\alpha, 1}\left(-(t-\tau)^{\alpha}\right)(f(\tau, u(\tau))-f(\tau, \widetilde{u}(\tau))) d \tau\right| \\
\leq & \mathcal{M} T\|u-\widetilde{u}\|_{\infty}+\mathcal{L} T\|u-\widetilde{u}\|_{\infty} .
\end{aligned}
$$

Hence $|(\mathcal{F} u)(t)-(\mathcal{F} \widetilde{u})(t)|<\epsilon_{1}$ whenever $\|u-\widetilde{u}\|_{\infty}<\delta_{1}, \delta_{1}<\frac{\epsilon_{1}}{(\mathcal{M}+\mathcal{L}) T}$. Which implies that $\mathcal{F}$ is relatively compact in $\mathcal{B}$.

Hence, by Schauder's fixed point theorem there exist at least one fixed point of $\mathcal{F}$ in $\mathcal{K}$.

Theorem 3.2. Assume that the following conditions are satisfied:

$\left(H_{2}\right)\|f(t, u)-f(t, v)\|_{\infty} \leq \mathcal{L}(t)\|u-v\|_{\infty}$,

$\left(H_{3}\right) \int_{0}^{t} a(\tau) d \tau \rightarrow 0$ as $t \rightarrow \infty$,

$\left(H_{4}\right) \int_{0}^{t} \mathcal{L}(\tau) d \tau \rightarrow 0$ as $t \rightarrow \infty$,

where $\mathcal{L}(t), a(t) \in \mathcal{L}^{1}[0, \infty)$ and $\mathcal{M}_{1}+\mathcal{M}_{2}<1$, where $\mathcal{M}_{1}=\sup _{t \in[0, \infty)} \int_{0}^{t} a(\tau) d \tau$, and $\mathcal{M}_{2}=\sup _{t \in[0, \infty)} \int_{0}^{t} \mathcal{L}(\tau) d \tau$. Then there exists a unique solution of (1.1) and $u(t) \rightarrow 0$ as $t \rightarrow \infty$. Moreover, the trivial solution of (1.1) is stable.

Proof. Setting $\mathcal{K}>\frac{\left|u_{0}\right|}{1-\mathcal{M}_{1}-\mathcal{M}_{2}}$. Define a set $\mathcal{E}=\left\{u \in C[\mathbb{R}, \mathbb{R}],\|u\|_{\infty} \leq \mathcal{K}\right.$ and $u(t) \rightarrow$ 0 as $t \rightarrow \infty\}$. Note that

$$
\begin{aligned}
|f(t, u)| & =|f(t, u)-f(t, 0)+f(t, 0)| \leq|f(t, u)-f(t, 0)|+|f(t, 0)| \\
& \leq \mathcal{L}\|u-0\|_{\infty}+0=\mathcal{L}\|u\|_{\infty} .
\end{aligned}
$$

Now, we define a mapping $\mathcal{F}$ on $\mathcal{E}$ as:

$$
\begin{aligned}
(\mathcal{F} u)(t)=u_{0} E_{\alpha, 1}\left(-t^{\alpha}\right)+\int_{0}^{t} E_{\alpha, 1}\left(-(t-\tau)^{\alpha}\right) f(\tau, u(\tau)) d \tau \\
\\
+\int_{0}^{t} E_{\alpha, 1}\left(-(t-\tau)^{\alpha}\right) a(\tau) u(\tau) d \tau .
\end{aligned}
$$

It is obvious that for $u \in \mathcal{E}, \mathcal{F} u$ is continuous.

First we prove that $\mathcal{F}$ maps $\mathcal{E}$ into itself.

$$
\|(\mathcal{F} u)(t)\|_{\infty} \leq\left|u_{0} E_{\alpha, 1}\left(-t^{\alpha}\right)\right|+\int_{0}^{t} E_{\alpha, 1}\left(-(t-\tau)^{\alpha}\right)|f(\tau, u(\tau))| d \tau
$$




$$
\begin{aligned}
& +\int_{0}^{t} E_{\alpha, 1}\left(-(t-\tau)^{\alpha}\right)|a(\tau)|\|u(\tau)\|_{\infty} d \tau \\
\leq & \left|u_{0}\right|+\mathcal{K} \int_{0}^{t} \mathcal{L}(\tau) d \tau+\mathcal{K} \int_{0}^{t} a(\tau) d \tau \\
\leq & \left|u_{0}\right|+\mathcal{K}\left(\mathcal{M}_{1}+\mathcal{M}_{2}\right) \leq \mathcal{K} .
\end{aligned}
$$

Hence $\mathcal{F}$ maps $\mathcal{E}$ into $\mathcal{E}$.

Next we show that $(\mathcal{F} u)(t) \rightarrow 0$ as $t \rightarrow \infty$.

Now, since $\lim _{t \rightarrow \infty} u_{0} E_{\alpha, 1}\left(-t^{\alpha}\right) \rightarrow 0$ as $t \rightarrow \infty$ and we have

$$
\begin{aligned}
\left|\int_{0}^{t} E_{\alpha, 1}\left(-(t-\tau)^{\alpha}\right) f(\tau, u(\tau)) d \tau\right| & \leq \int_{0}^{t} E_{\alpha, 1}\left(-(t-\tau)^{\alpha}\right)|f(\tau, u(\tau))| d \tau \\
& \leq \int_{0}^{t} E_{\alpha, 1}\left(-(t-\tau)^{\alpha}\right) \mathcal{L}(\tau)\|u\|_{\infty} d \tau \\
& \leq \mathcal{K} \int_{0}^{t} E_{\alpha, 1}\left(-(t-\tau)^{\alpha}\right) \mathcal{L}(\tau) d \tau \\
& \leq \mathcal{K} \int_{0}^{t} \mathcal{L}(\tau) d \tau \leq \mathcal{K} \epsilon, \epsilon>0
\end{aligned}
$$

Thus $\left|\int_{0}^{t} E_{\alpha, 1}\left(-(t-\tau)^{\alpha}\right) f(\tau, u(\tau)) d \tau\right| \rightarrow 0$ as $t \rightarrow \infty$. And

$$
\begin{aligned}
\left|\int_{0}^{t} E_{\alpha, 1}\left(-(t-\tau)^{\alpha}\right) a(\tau) u(\tau) d \tau\right| & \leq \int_{0}^{t} E_{\alpha, 1}\left(-(t-\tau)^{\alpha}\right)|a(\tau)||u(\tau)| d \tau \\
& \leq \mathcal{K} \int_{0}^{t} E_{\alpha, 1}\left(-(t-\tau)^{\alpha}\right)|a(\tau)| d \tau \\
& \leq \mathcal{K} \int_{0}^{t} a(\tau) d \tau \leq \mathcal{K} \epsilon, \epsilon>0
\end{aligned}
$$

implies $\left|\int_{0}^{t} E_{\alpha, 1}\left(-(t-\tau)^{\alpha}\right) a(\tau) u(\tau) d \tau\right| \rightarrow 0$ as $t \rightarrow \infty$.

Hence $(\mathcal{F} u)(t) \rightarrow 0$ as $t \rightarrow \infty$.

Next, we prove that $\mathcal{F}$ is a contraction mapping.

$$
\begin{aligned}
|(\mathcal{F} u)(t)-(\mathcal{F} v)(t)| & \leq \int_{0}^{t} E_{\alpha, 1}\left(-(t-\tau)^{\alpha}[a(\tau)+\mathcal{L}(\tau)]\|u-v\|_{\infty} d \tau\right. \\
& =\left(\mathcal{M}_{1}+\mathcal{M}_{2}\right)\|u-v\|_{\infty} .
\end{aligned}
$$

Since $\mathcal{M}_{1}+\mathcal{M}_{2}<1$. Thus $\mathcal{F}$ is contraction. Hence by contraction mapping principle (1.1) has a unique solution. The stability of trivial solution of (1.1) follows just by replacing $\mathcal{K}$ by $\epsilon$. This completes the proof.

Theorem 3.3. Let $u$ be the solution of (1.1), and $v$ be the solution of the following initial value problem

$$
\begin{aligned}
{ }^{c} D_{0, t}^{\alpha} v(t)+v(t) & =a(t) v(t)+f(t, v(t)), \quad 0<\alpha \leq 1, \\
v(0) & =v_{0} .
\end{aligned}
$$


Moreover, let for any very small $\epsilon>0, \delta=\left(1-\mathcal{M}_{1}-\mathcal{M}_{2}\right) \epsilon$. Then under the conditions of Theorem 3.2, we have

$$
\|u-v\|_{\infty}=\mathcal{O}\left(\frac{\left|u_{0}-v_{0}\right|}{1-\mathcal{M}_{1}-\mathcal{M}_{2}}\right) .
$$

Proof. Since $u$ is solution of (1.1) and $v$ is solution of (3.2) satisfying the initial condition $v(0)=v_{0}$. Then

$$
\begin{aligned}
\|u-v\|_{\infty} \leq & \left|u_{0}-v_{0}\right| E_{\alpha, 1}\left(-t^{\alpha}\right)+\left|\int_{0}^{t} E_{\alpha, 1}\left(-(t-\tau)^{\alpha}\right)(f(\tau, u(\tau))-f(\tau, v(\tau))) d \tau\right| \\
& +\left|\int_{0}^{t} E_{\alpha, 1}\left(-(t-\tau)^{\alpha}\right)(a(\tau) u(\tau)-a(\tau) v(\tau)) d \tau\right| \\
\leq & \left|u_{0}-v_{0}\right| E_{\alpha, 1}\left(-t^{\alpha}\right)+\int_{0}^{t} E_{\alpha, 1}\left(-(t-\tau)^{\alpha}\right)|f(\tau, u(\tau))-f(\tau, v(\tau))| d \tau \\
& \quad+\int_{0}^{t} E_{\alpha, 1}\left(-(t-\tau)^{\alpha}\right)|a(\tau) u(\tau)-a(\tau) v(\tau)| d \tau \\
\leq & \left|u_{0}-v_{0}\right|+\int_{0}^{t}\|f(\tau, u(\tau))-f(\tau, v(\tau))\|_{\infty} d \tau+\int_{0}^{t} a(\tau)|u(\tau)-v(\tau)| d \tau \\
\leq & \left|u_{0}-v_{0}\right|+\int_{0}^{t} \mathcal{L}(\tau)\|u(\tau)-v(\tau)\|_{\infty} d \tau+\int_{0}^{t} a(\tau)|u(\tau)-v(\tau)| d \tau \\
\leq & \left|u_{0}-v_{0}\right|+\|u(\tau)-v(\tau)\|_{\infty} \int_{0}^{t} \mathcal{L}(\tau) d \tau+\|u(\tau)-v(\tau)\|_{\infty} \int_{0}^{t} a(\tau) d \tau \\
\leq & \left|u_{0}-v_{0}\right|+\mathcal{M}_{2}\|u(\tau)-v(\tau)\|_{\infty}+\mathcal{M}_{1}\|u(\tau)-v(\tau)\|_{\infty},
\end{aligned}
$$

implies $\|u-v\|_{\infty}=\mathcal{O}\left(\frac{\left|u_{0}-v_{0}\right|}{1-\mathcal{M}_{1}-\mathcal{M}_{2}}\right)$ as desired.

Remark 3.4. From Theorem 3.2 and Theorem 3.3, it is clear that solution of (1.1) is asymptotically stable.

Theorem 3.5. Suppose that $\left(H_{1}\right)$ holds and there exist constants $\rho_{1}, \rho_{2}$ such that $0<\rho_{1}<1, \rho_{2} \in\left(0,1-\rho_{1}\right)$ and a uniformly continuous function $f$ on the compact set $\left[0, \chi_{1}\right] \times \mathcal{M}(\epsilon)$ so that for given $\epsilon_{1}>0$, exists a $\delta_{1}>0$ such that $\left(H_{5}\right)|f(t, u)-f(t, v)|<\frac{\epsilon_{1}}{\chi_{1}}$ whenever $\|u-v\|_{\infty}<\delta_{1}$.

Then the solution $u \equiv 0$ of (1.1) is stable in Banach space $\mathcal{B}$.

Proof. Let $0<\delta<\left(1-\rho_{1}-\rho_{2}\right) \epsilon$. Consider the non-empty closed convex subset $\mathcal{M}(\epsilon) \subseteq \mathcal{B}$, and define two mappings $\mathcal{F}_{1}, \mathcal{F}_{2}$ on $\mathcal{M}(\epsilon)$ for $t \geq 0$, as follows:

$$
\begin{aligned}
& \mathcal{F}_{1} u(t)=\int_{0}^{t} E_{\alpha, 1}\left(-(t-\tau)^{\alpha}\right) f(\tau, u(\tau)) d \tau \\
& \mathcal{F}_{2} u(t)=x_{0} E_{\alpha, 1}\left(-t^{\alpha}\right)+\int_{0}^{t} E_{\alpha, 1}\left(-(t-\tau)^{\alpha}\right) a(\tau) u(\tau) d \tau .
\end{aligned}
$$


For $u \in \mathcal{M}(\epsilon)$ and $t \in\left[0, \chi_{1}\right]$, we obtain

$$
\begin{aligned}
\left|\mathcal{F}_{1} u(t)\right| & =\left|\int_{0}^{t} E_{\alpha, 1}\left(-(t-\tau)^{\alpha}\right) f(\tau, u(\tau)) d \tau\right| \leq\|f\|_{\infty}|t| \leq\|f\|_{\infty} \chi_{1}<\infty, \\
\left|\mathcal{F}_{2} u(t)\right| & =\left|u_{0} E_{\alpha, 1}\left(-t^{\alpha}\right)+\int_{0}^{t} E_{\alpha, 1}\left(-(t-\tau)^{\alpha}\right) a(\tau) u(\tau) d \tau\right| \\
& \leq\left|u_{0}\right|+\rho_{2}|| u \|_{\infty}<\infty
\end{aligned}
$$

Then $\mathcal{F}_{1} \mathcal{M}(\epsilon) \subseteq \mathcal{B}$ and $\mathcal{F}_{2} \mathcal{M}(\epsilon) \subseteq \mathcal{B}$. Now we prove the existence of at least one fixed point of the operator $\mathcal{F}_{1}+\mathcal{F}_{2}$ in $\mathcal{M}(\epsilon)$.

Firstly, we prove that $\mathcal{F}_{1} u+\mathcal{F}_{2} v \in \mathcal{M}(\epsilon)$ for all $u, v \in \mathcal{M}(\epsilon)$.

For any $u, v \in \mathcal{M}(\epsilon)$, from $\left(H_{1}\right)$ we get that

$$
\begin{aligned}
\sup _{t \geq 0}\left|\mathcal{F}_{1} u+\mathcal{F}_{2} v\right| & =\sup _{t \geq 0}\left\{\mid \int_{0}^{t} E_{\alpha, 1}\left(-(t-\tau)^{\alpha}\right) f(\tau, u(\tau)) d \tau\right. \\
& \left.+u_{0} E_{\alpha, 1}\left(-t^{\alpha}\right)+\int_{0}^{t} E_{\alpha, 1}\left(-(t-\tau)^{\alpha}\right) a(\tau) u(\tau) d \tau \mid\right\} \\
& \leq \rho_{1} \epsilon+\delta+\rho_{2} \epsilon \leq \epsilon
\end{aligned}
$$

which implies $\mathcal{F}_{1} u+\mathcal{F}_{2} v \in \mathcal{M}(\epsilon)$ for all $u, v \in \mathcal{M}(\epsilon)$.

Secondly, we prove that $\mathcal{F}_{1} \mathcal{M}(\epsilon)$ is relatively compact in $\mathcal{B}$.

Taking $0 \leq t_{1} \leq t_{2} \leq t$, we have

$$
\begin{aligned}
\left|\mathcal{F}_{1} u\left(t_{1}\right)-\mathcal{F}_{1} u\left(t_{2}\right)\right|= & \mid \int_{0}^{t_{1}} E_{\alpha, 1}\left(-\left(t_{1}-\tau\right)^{\alpha}\right) f(\tau, u(\tau)) d \tau \\
& -\int_{0}^{t_{2}} E_{\alpha, 1}\left(-\left(t_{2}-\tau\right)^{\alpha}\right) f(\tau, u(\tau)) d \tau \mid \\
\leq & \|f\|_{\infty}\left(t_{2}-t_{1}\right) \rightarrow 0,
\end{aligned}
$$

as $t_{1} \rightarrow t_{2}$. Now, let $u, \tilde{u} \in \mathcal{M}(\epsilon)$ such that $\|u-\tilde{u}\|_{\infty}<\delta_{1}$. Then, in view of $\left(H_{5}\right)$, we obtain

$$
|f(t, u)-f(t, \tilde{u})|<\frac{\epsilon_{1}}{\chi_{1}} \quad \text { for all } t \in\left[0, \chi_{1}\right]
$$

Hence,

$$
\begin{aligned}
\left|\left(\mathcal{F}_{1} u\right)(t)-\left(\mathcal{F}_{1} \tilde{u}\right)(t)\right|= & \mid \int_{0}^{t} E_{\alpha, 1}\left(-(t-\tau)^{\alpha}\right) f(\tau, u(\tau)) d \tau \\
& -\int_{0}^{t} E_{\alpha, 1}\left(-(t-\tau)^{\alpha}\right) f(\tau, \tilde{u}(\tau)) d \tau \mid \\
\leq & \frac{\epsilon_{1}}{\chi_{1}}|t|=\epsilon_{1},
\end{aligned}
$$

which proves our required conclusion. 
Thirdly, we argue that $\mathcal{F}_{2}: \mathcal{M}(\epsilon) \rightarrow \mathcal{B}$ is a contraction mapping.

For any $u, v \in \mathcal{M}(\epsilon)$, from $\left(H_{1}\right)$, we get

$$
\begin{aligned}
\sup _{t \geq 0}\left|\mathcal{F}_{2} u(t)-\mathcal{F}_{2} v(t)\right| & =\sup _{t \geq 0}\left\{\left|\int_{0}^{t} E_{\alpha, 1}\left(-(t-\tau)^{\alpha}\right) a(\tau)(u(\tau)-v(\tau)) d \tau\right|\right\} \\
& \leq \rho_{2}\|u-v\|_{\infty}<\|u-v\|_{\infty} .
\end{aligned}
$$

Hence, the operator $\mathcal{F}_{1}+\mathcal{F}_{2}$ has at least one fixed point in $\mathcal{M}(\epsilon)$.

Finally, for any $\epsilon_{2}>0$, if $0<\delta_{2}<\left(1-\rho_{1}-\rho_{2}\right) \epsilon_{2}$, then $\left|u_{0}\right|<\delta_{2}$ implies that

$$
\begin{aligned}
\|u\|_{\infty}= & \sup _{t \geq 0}\left\{\mid u_{0} E_{\alpha, 1}\left(-t^{\alpha}\right)+\int_{0}^{t} E_{\alpha, 1}\left(-(t-\tau)^{\alpha}\right) a(\tau) u(\tau) d \tau\right. \\
& \left.+\int_{0}^{t} E_{\alpha, 1}\left(-(t-\tau)^{\alpha}\right) f(\tau, u(\tau)) d \tau \mid\right\} \\
\leq & \epsilon_{2} .
\end{aligned}
$$

Thus, the solution of (1.1) is stable in Banach space $\mathcal{B}$.

Theorem 3.6. Assume that all the conditions of Theorem 3.5 are fulfilled. And there exists a function $\phi_{\rho}(t) \in \mathcal{L}^{1}\left[0, \chi_{1}\right], \phi_{\rho}(t)>0$ for any $\rho>0$, such that $|\tau| \leq \rho$ implies

$$
|f(t, \tau)| \leq \phi_{\rho}(t), \quad t \in[0, \infty) .
$$

Then the zero solution of (1.1) is asymptotically stable.

Proof. Stability of zero solution is guaranteed by Theorem 3.5. Now, we prove that the zero solution of (1.1) is attractive.

Defining

$$
\mathcal{M}_{*}(\rho)=\left\{u: u \in \mathcal{M}(\rho), \lim _{t \rightarrow \infty} u(t)=0\right\} \text { for any } \rho>0 \text {. }
$$

For this we will show that, for any $u, v \in \mathcal{M}_{*}(\rho), \quad \mathcal{F}_{1} u+\mathcal{F}_{2} v \in \mathcal{M}_{*}(\rho)$ that is, as $t \rightarrow \infty, \quad \mathcal{F}_{1} u(t)+\mathcal{F}_{2} v(t) \rightarrow 0$, where

$$
\begin{aligned}
\mathcal{F}_{1} u(t)+\mathcal{F}_{2} v(t)=u_{0} E_{\alpha, 1}\left(-t^{\alpha}\right)+\int_{0}^{t} E_{\alpha, 1}(-(t & \left.-\tau)^{\alpha}\right) f(\tau, u(\tau)) d \tau \\
& +\int_{0}^{t} E_{\alpha, 1}\left(-(t-\tau)^{\alpha}\right) a(\tau) u(\tau) d \tau
\end{aligned}
$$

In fact, for $u, v \in \mathcal{M}_{*}(\rho)$, stem from the similar argument used in the proof of the second step of Theorem 3.5, and we obtain our required conclusion by $(2.2),\left(H_{1}\right)$ and by the hypothesis that $\phi_{\rho}(t) \in \mathcal{L}^{1}\left[0, \chi_{1}\right]$.

Finally, we give an example to illustrate our result. 
Example 3.7. Consider the following non-linear fractional differential equation

$$
\begin{aligned}
{ }^{c} D_{0, t}^{\frac{1}{2}} u(t)+u(t) & =\frac{1}{3} u \cos t+u^{2} e^{-\left(u^{2}+t^{2}\right)}, t \geq 0 \\
u(0) & =u_{0},
\end{aligned}
$$

where $u_{0} \in \mathbb{R}, a(t)=\frac{1}{3} \cos t, f(t, u)=u^{2} e^{-\left(u^{2}+t^{2}\right)}$. Obviously, $f(t, 0) \equiv 0$. Let $\rho_{1}=\frac{1}{2}$ and $\rho_{2}=\frac{1}{3}$, so we get $\sup _{t \geq 0} \int_{0}^{t} a(\tau) d \tau \leq \frac{1}{3}$, that is assumption $\left(H_{1}\right)$ is satisfied. Now by computations we have

$$
\|f\|_{\infty}=\sup _{t \geq 0}|f(t, u)|=\frac{1}{e}, \quad \chi_{1}=\frac{\rho_{1} \epsilon}{\|f\|_{\infty}}=\frac{\epsilon}{2 e}, \epsilon>0 .
$$

And clearly $f$ is uniformly continuous on the compact set $\left[0, \chi_{1}\right] \times \mathcal{M}(\epsilon)$, where $M(\epsilon)=$ $\left\{u: u \in B,\|u\|_{\infty}<\epsilon\right\}$. Further we choose $\epsilon_{1}=\epsilon>0$, and found $\delta_{1}=\frac{2 e}{\mathcal{M}_{1}}>0$, for some constant $\mathcal{M}_{1}>0$, such that $|f(t, u)-f(t, v)| \leq|u+v||u-v| \leq \mathcal{M}_{1}|u-v|<2 e$, whenever $|u-v|<\delta_{1}$.

Since Theorem 3.5 fulfilled. Hence the trivial solution of (1.1) is stable in Banach space $\mathcal{B}$.

\section{REFERENCES}

[1] R. Hilfer, Applications of Fractional Calculus in Physics, World Scientific, 2000.

[2] K. B. Oldham, Fractional differential equations in electrochemistry, Advances in Engineering Software, 41, (2010) 9-12.

[3] R. L. Magin, Fractional calculus models of complex dynamics in biological tissues, Computers and Mathematics with Applications, 59, (2010) 1586-1593.

[4] M. D. Ortigueira, Fractional Calculus for Scientists and Engineers, Springer, 2011.

[5] J. Singh, D. Kumar, J. J. Nieto, Analysis of an el nino-southern oscillation model with a new fractional derivative, Chaos, Solitons and Fractals, 99, (2017) 109115 .

[6] Y. Tian, J. J. Nieto, The applications of critical-point theory discontinuous fractional-order differential equations, Proceedings of the Edinburgh Mathematical Society, 2014, (2014) 1-31, DOI: 10.1017/S001309151600050X.

[7] J. J. Nieto, Solvability of an implicit fractional integral equation via a measure of noncompactness argument, Acta Mathematica Scientia, 37, (2017) 195-204.

[8] X. Gao, J. Yu, Chaos in the fractional order periodically forced complex Duffing oscillators, Chaos, Solitons and Fractals, 24, (2005) 1097-1104. 
[9] J. R. Wanga, Y. Zhoub, M. Feckan, Nonlinear impulsive problems for fractional differential equations and Ulam stability, Computers and Mathematics with Applications, 64, (2012) 3389-3405.

[10] Z. M. Odibat, Analytic study on linear systems of fractional differential equations, Computers and Mathematics with Applications, 59, (2010) 1171-1183.

[11] Bashir Ahmada, S. Sivasundaram, Dynamics and stability of impulsive hybrid setvalued integro-differential equations with delay, Nonlinear Analysis, 65, (2006) 2082-2093.

[12] S. Abbas, E. Alaidarous, M. Benchohra, J. J. Nieto, Existence and stability of solutions for Hadamard-Stieltjes fractional integral equations, Discrete Dynamics in Nature and Society, 2015, (2015) DOI: 10.1155/2015/317094.

[13] M. D. la Sen, Total stability properties based on fixed point theory for a class of hybrid dynamic systems, Fixed Point Theory and Applications, 2009 (2009) DOI: $10.1155 / 2009 / 826438$.

[14] J. O. Alzabut, J. J. Nieto, G. Tr. Stamov, Existence and exponential stability of positive almost periodic solutions for a model of hematopoiesis, Boundary Value Problems, 2009 (2009) DOI: 10.1155/2009/127510.

[15] G. M. Zaslavsky, A. A. Stanislavsky, M. Edelman, Chaotic and pseudochaotic attractors of perturbed fractional oscillator, Chaos 16, (2006) 013102.

[16] E. Ahmed, A. M. A. El-Sayed, H. A. A. El-Saka, Equilibrium points, stability and numerical solutions of fractional-order predatorprey and rabies models, Journal of Mathematical Analysis and Applications, 325, (2007) 542-553.

[17] T.A. Burton, B. Zhang, Fractional equations and generalizations of Schaefers and Krasnoselskiis fixed point theorems, Nonlinear Analysis: Theory, Methods and Applications, 75, (2012) 6485-6495.

[18] F. Ge, C. Kou, Stability analysis by Krasnoselskii's fixed point theorem for nonlinear fractional differential equations, Applied Mathematics and Computation, 257, (2015) 308-316.

[19] I. Podlubny, Fractional Differential Equations, Academic Press, San Diego, 1999.

[20] M. Concezzi, R. Spigler, Some analytical and numerical properties of the MittagLeffler functions, Fractional Calculus and Applied Analysis 18, (2015) 64-94.

[21] D. Qian, C. Li, R. P. Agarwal, P. J. Wong, Stability analysis of fractional differential system with Riemann-Liouville derivative, Mathematical and Computer Modelling, 52, (2010) 862-874. 